\title{
Perfil da violência sexual contra meninos, SINAN, 2009-2017, Brasil
}

\author{
Profile of notification sexual violence against boys, sinan 2009-2017, Brazil \\ Perfil de la notificación de la violencia contra los niños, sinan 2009-2017, Brasil
}

\author{
Priscila Arruda da Silva \\ ORCID: https://orcid.org/0000-0002-5359-8646 \\ Universidade Federal do Rio Grande, Brasil \\ E-mail:patitaarruda@yahoo.com.br \\ Simone Algeri \\ ORCID: https://orcid.org/0000-0002-3152-0944 \\ Universidade Federal do Rio Grande do Sul, Brasil \\ E-mail: simone.salgeri@gmail.com \\ Michele Peixoto da Silva \\ ORCID: https://orcid.org/0000-0002-4350-0588 \\ Prefeitura Municipal do Rio Grande, Brasil \\ E-mail:chele.p@hotmail.com \\ Mariana Lima Corrêa \\ ORCID: https://orcid.org/0000-0003-2373-3584 \\ Centro de Pesquisas Epidemiológicas, Brasil \\ E-mail: mari_lima_correa@hotmail.com \\ Maria da Penha da Rosa Nunes \\ ORCID: https://orcid.org/0000-0003-2117-1581 \\ Prefeitura Municipal do Rio Grande, Brasil \\ E-mail: mariadapenha23@hotmail.com
}

\section{Resumo}

Este estudo analisou o perfil epidemiológico das notificações de violência sexual contra crianças do sexo masculino, no Brasil, com base no Sistema de Informação de Agravos de Notificação (SINAN), entre 2009 e 2017. Foram consideradas todas as notificações de violência sexual contra meninos, de acordo com as variáveis: idade, cor da pele, local de ocorrência, relação com a vítima e reincidência. Os dados foram extraídos e apresentados em tabelas na forma de frequência absoluta e percentual. No período analisado, foram registradas 17.451 notificações de violência sexual contra meninos. O número de notificações aumentou em quase 3,5 vezes, passando de 790 em 2009 para 2.750 em 2017. A faixa etária de 5 a 9 anos teve maior número de notificações (10.836 casos), e a maior prevalência de notificações foi na região Sudeste $(42,5 \%)$. As informações produzidas neste estudo poderão ser úteis para o planejamento de ações ou políticas públicas voltadas a crianças do sexo masculino, vítimas de violência sexual.

Palavras-chave: Delitos sexuais; Notificação de abuso; Crianças; Defesa da criança e do adolescente; Sistemas de informação.

\begin{abstract}
This study analyzed the epidemiological profile of notification of sexual violence against male children in Brazil, based on the Sistema de Informação de Agravos de Notificação (SINAN), between 2009 and 2017. All notifications of sexual violence against boys were considered according with the variables age, skin color, place of occurrence, relationship with the victim and recurrence. Data were extracted and presented in tables as absolute frequency and percentage. During the analyzed period, there were 17,451 reports of sexual violence against boys. The number of notifications increased by almost 3.5 times, from 790 in 2009 to 2,750 in 2017. The 5-9 years age group had the highest number of notifications (10,836 cases), and the highest prevalence of notifications was in the Southeast region $(42.5 \%)$. The information produced in this study may be useful for planning actions or public policies aimed at male children victims of sexual violence.
\end{abstract}

Keywords: Sex offences; Mandatory reporting; Children; Child advocacy; Information system.

\section{Resumen}

Este estudio analizó el perfil epidemiológico de la notificación de la violencia sexual contra niños varones en Brasil, basado en el Sistema de Información de Enfermedades Notificables (SINAN), entre 2009 y 2017. Se consideraron todos los informes de violencia sexual contra niños, de acuerdo con las variables: edad, color de piel, lugar de ocurrencia, relación con la víctima y recurrencia. Los dados fueron extraídos y presentados en tablas en forma de frecuencia absoluta y porcentaje. En el periodo analizado, se registraron 17.451 denuncias de violencia sexual contra niños. El número de notificaciones aumentó casi 3,5 veces, de 790 en 2009 a 2.750 en 2017. El grupo de edad de 5 a 9 
años tuvo un mayor número de notificaciones (10.836 casos), y la mayor prevalencia de notificaciones se produjo en la región sudeste. (42,5\%). La información producida en este estudio puede ser útil para planificar acciones de las políticas públicas dirigidas a niños varones, víctimas de violencia sexual.

Palabras clave: Delitos sexuales; Notificación de abuso; Niños; Defensa de niños y adolescentes; Sistemas de información.

\section{Introdução}

A violência sexual infantil é considerada como um problema grave de saúde pública, face as consequências ao longo do ciclo vital (Oliveira et al, 2014; Platt et al, 2018; Reis \& Parra, 2018). Caracterizada como uma demonstração de brutalidade extrema, é uma modalidade de violência em que a vítima tem seu corpo, sua vontade e seus direitos negados (Dong et al, 2003; Sanches et al; 2019).

Representada por duas categorias, em que a vítima é utilizada pelo agressor, mediante uma relação de dominação, na busca da sua própria satisfação sexual e de forma comercial e mercantil, se tornam vítimas de um sistema de exploração comercial de sua sexualidade, a violência sexual tem representado na atualidade, sério comprometimento na saúde e desenvolvimento infanto-juvenil (Ellery, Correa \& Gadelha, 2008; Oliveira et al, 2014).

Levar publicamente uma situação de violência sexual não é tarefa fácil, visto o temor das repercussões que essa notícia pode gerar na família, na sociedade e para os profissionais que trabalham com crianças e adolescentes. Frequentemente, as vítimas sentem-se culpabilizadas por terem provocado a agressão; além do sentimento de culpa, também, por não denunciarem a violência sofrida, principalmente quando são crianças e adolescentes (Carneiro \& Cabral, 2010; Galo et al 2017; Lopez \& Lefevre, 2019).

Apesar do impacto emocional que aparentemente este tipo de violência provoca na sociedade, estudo de revisão sobre abuso sexual contra crianças apontou que sua ocorrência se reflete em uma resposta social ambígua. Diferentemente do que se pensa, a vítima tem enfrentado preconceitos da sociedade e, em algumas situações, dos próprios membros da família que deveriam apoiá-la (Martins \& Jorge, 2010).

Nota-se, então, que a temática carece de maior visibilidade social, a fim de que vítimas, profissionais e sociedade em geral possam percebê-la como um problema de saúde coletiva. A complexidade do tema exige um olhar mais direcionado a crianças em desenvolvimento, pois embora a violência permeie todas as faixas etárias é na primeira infância que o impacto é maior. Isto reforça a seriedade do problema da violência, já que essas situações não se restringem apenas àquela criança, no momento que vivencia a experiência de ser violado em seus direitos, mas pode se expandir num ciclo entre gerações (Lira et al, 2017).

Considerando que a violência sexual na população masculina é menos noticiada e observada na literatura, quando comparados a vítimas do sexo feminino, este estudo busca, analisar o perfil da violência sexual contra crianças do sexo masculino, no Brasil, com base no Sistema de Informação de Agravos de Notificação (SINAN), entre 2009 e 2017.

\section{Metodologia}

Trata-se de um estudo descritivo sobre o perfil epidemiológico das violências sexuais contra crianças do sexo masculino, notificados no Sistema de Informação de Agravos de Notificação (SINAN), no período de 2009 a 2017. O recorte temporal foi definido em função do período em que a notificação de violências e acidentes passou a integrar o sistema (2009), permitindo a sua consolidação em todo território brasileiro, no que diz respeito à padronização de coleta e envio dos dados.

Este estudo delimita como crianças os indivíduos com idade entre zero e 9 anos, conforme a convenção elaborada pela Organização Mundial da Saúde (OMS) e adotada pelo Ministério da Saúde (Brasil, 2018). 
Os dados utilizados neste estudo foram extraídos do Sistema de Informação de Agravos de Notificação (SINAN), a partir das fichas de notificação individual de violência interpessoal/autoprovocada. Incluem-se como violência sexual os casos de assédio, estupro, atentado violento ao pudor, pornografia infantil e exploração sexual contra crianças do sexo masculino. Como critérios de exclusão, foram considerados os dados de crianças do sexo feminino, que não se encontram na faixa etária estipulada.

A coleta dos dados ocorreu em fevereiro de 2020, sendo realizada uma análise descritiva das características sociodemográficas das vítimas de violência sexual (idade, raça/cor da pele, região de residência), das características da violência (reincidência, local de ocorrência e tipo de violência sexual) e das características do provável autor da violência sexual (sexo do agressor, vínculo com a vítima).

Embora seja um estudo com dados secundário, sem envolvimento de seres humanos, este faz parte de um projeto maior, com certificação ética, aprovado com o parecer CAAE: 49775415.8.0000.5324.

\section{Resultados}

No período de 2009 a 2017, foram notificados no Sinan 17.451 casos de violência contra crianças do sexo masculino. Comparando-se os anos de 2009 e 2017, observa-se um aumento de 3,5 vezes no número de notificações de violência sexual contra meninos, passando de 790 casos em 2009, para 2750 em 2017, evidenciando-se assim, um aumento significativo de registros de violência sexual contra meninos (Figura 1).

Figura 1: Número de notificações de violência sexual contra meninos, segundo ano de notificação, Brasil 2009-2017.

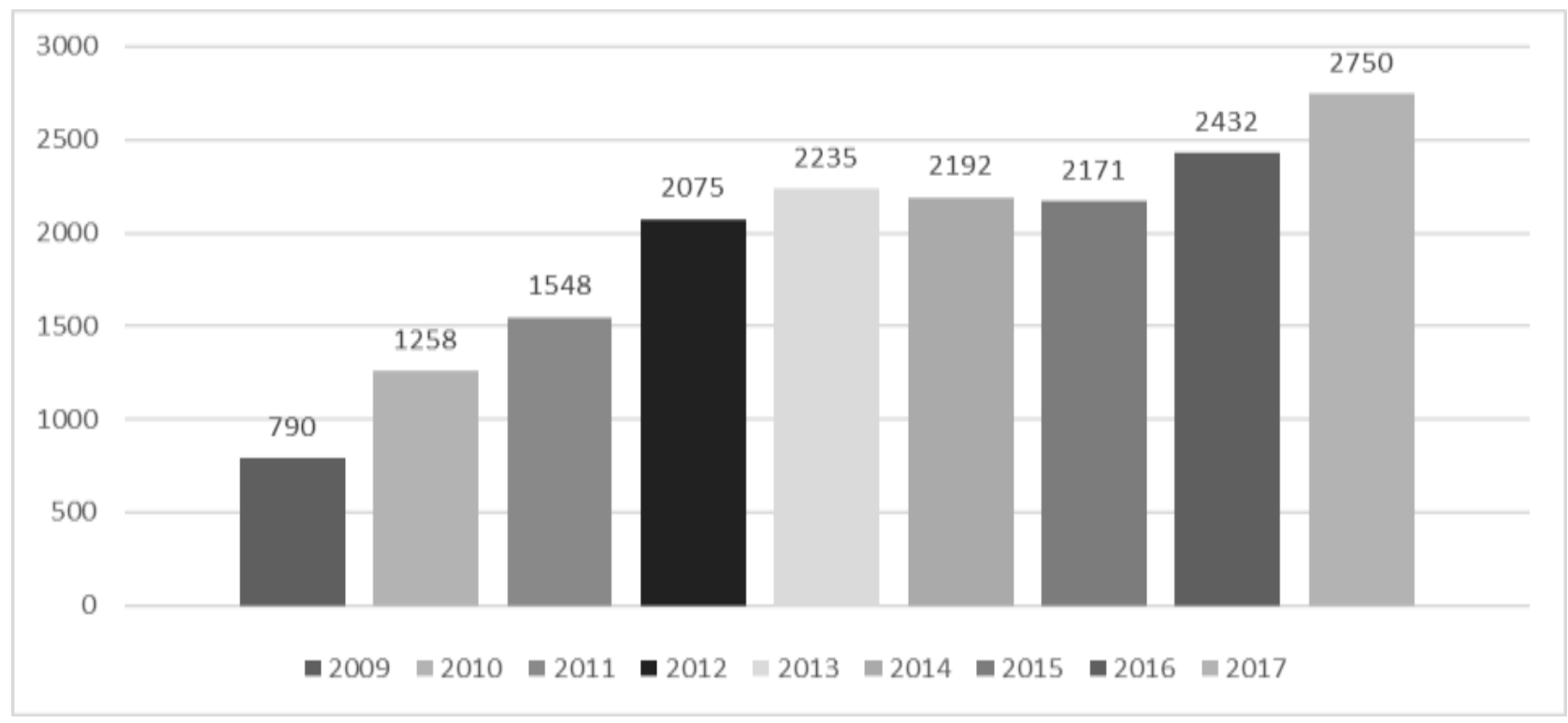

Fonte: Sinan/Ministério da Saúde.

\section{Perfil das notificações de violência sexual contra crianças do sexo masculino no Brasil}

\section{Características sociodemográficas das vítimas}

A avaliação das características sociodemográficas de crianças do sexo masculino vítimas de violência sexual mostrou que do total de 17.451 notificações, 62,1\% correspondiam à faixa etária entre 5 e 9 anos e 44,2\% à raça/cor da pele preta/parda. As notificações se concentraram na região Sudeste (42,5\%), Sul (23,6\%) e Norte (13\%). (Tabela 1). 
Tabela 1: Características sociodemográficas de crianças do sexo masculinos vítimas de violência sexual notificados no SINAN, Brasil, 2009 - 2017.

\begin{tabular}{|l|c|c|}
\hline Variáveis & $\%$ & $\mathrm{n}$ \\
\hline Faixa Etária (anos) & & \\
\hline$<1$ & 2,6 & 459 \\
\hline 1 a 4 & 35,3 & 6156 \\
\hline 5 a 9 & 62,1 & 10836 \\
\hline Raça/cor da pele & & \\
\hline Branca & 40,4 & 7059 \\
\hline Preta/Parda & 44,2 & 7683 \\
\hline Amarela/outra & 0,9 & 169 \\
\hline Ign/branco & 14,5 & 2540 \\
\hline Região de residência & & \\
\hline Norte & 13 & 2261 \\
\hline Nordeste & 10,3 & 1807 \\
\hline Sudeste & 42,5 & 7419 \\
\hline Sul & 23,6 & 4127 \\
\hline Centro-oeste & 10,5 & 1835 \\
\hline Ign/exterior & 0,1 & 2 \\
\hline Total & $\mathbf{1 0 0}$ & $\mathbf{1 7 4 5 1}$ \\
\hline
\end{tabular}

Fonte: Autores.

\section{Características da violência sexual}

A avaliação das características da violência sexual contra crianças do sexo masculino mostrou que $33,2 \%$ dos eventos tiveram caráter de repetição, 63,5\% ocorreram na residência da vítima e 52,6\% foram notificados como estupro (Tabela 2).

Tabela 2: Características da violência sexual contra crianças do sexo masculino, notificadas no Sinan, Brasil, 2009-2017.

\begin{tabular}{|c|c|c|}
\hline Variáveis & $\%$ & $\mathrm{n}$ \\
\hline \multicolumn{3}{|l|}{ Reincidência } \\
\hline Sim & 33,2 & 5792 \\
\hline Não & 31,8 & 5552 \\
\hline Ignorado & 35,0 & 6107 \\
\hline \multicolumn{3}{|l|}{ Local de ocorrência } \\
\hline Residência & 63,5 & 11075 \\
\hline Habitação coletiva & 1,1 & 199 \\
\hline Escola & 7,0 & 1215 \\
\hline Local de prática esportiva & 0,7 & 119 \\
\hline Bar & 0,3 & 51 \\
\hline Via pública & 4,3 & 757 \\
\hline Comércio & 0,4 & 71 \\
\hline Indústria & 0,3 & 57 \\
\hline Outros & 10 & 1757 \\
\hline Ign/Branco & 12,3 & 2150 \\
\hline \multicolumn{3}{|l|}{ Tipo de violência sexual ${ }^{\mathrm{a}}$} \\
\hline Assédio sexual & 22,1 & 4256 \\
\hline Estupro & 52,6 & 10.114 \\
\hline Pornografia infantil & 3,7 & 704 \\
\hline Exploração sexual & 2,9 & 563 \\
\hline Atentado violento ao pudor & 12,6 & 2425 \\
\hline Outros & 6,0 & 1166 \\
\hline Total & 100 & 17451 \\
\hline
\end{tabular}

${ }^{\mathrm{a}}$ variável com n maior $=19.228$. Fonte: Sinan/Ministério da Saúde. 


\section{Características do possível autor da violência}

A avaliação das características do possível autor da violência sexual contra crianças mostrou que, em 19,3\% das notificações, o agressor era do sexo masculino e 34,8\% tinham vínculo de amizade/conhecimento da vítima (Tabela 3).

Tabela 3: Características do possível autor da violência sexual contra crianças do sexo masculino, notificadas no Sinan, Brasil, 2009-2017.

\begin{tabular}{|c|c|c|}
\hline Variáveis & $\%$ & $\mathrm{n}$ \\
\hline \multicolumn{3}{|l|}{ Sexo do autor } \\
\hline Masculino & 19,3 & 3375 \\
\hline Feminino & 3,7 & 659 \\
\hline Ambos os sexos & 19,2 & 3352 \\
\hline Ign/branco & 57,6 & 10065 \\
\hline \multicolumn{3}{|c|}{ Vínculo do autor com a vítima ${ }^{a}$} \\
\hline Pai & 12,9 & 2258 \\
\hline Mãe & 3,4 & 604 \\
\hline Padrasto & 6,4 & 1117 \\
\hline Madrasta & 0,3 & 55 \\
\hline Irmão (a) & 4,8 & 844 \\
\hline Amigos/conhecidos & 34,8 & 6084 \\
\hline Desconhecido(a) & 12,4 & 2172 \\
\hline Cuidador(a) & 1,9 & 336 \\
\hline Outros vínculos & 22,8 & 3981 \\
\hline Total & 100 & 17451 \\
\hline
\end{tabular}

Fonte: Autores.

\section{Discussão}

A análise da violência sexual contra crianças do sexo masculino, no Brasil, permite revelar que os dados sobre o tema ainda são incipientes. Pode-se notar, através dos dados apresentados neste estudo, um aumento gradativo nas notificações de violência ao longo dos anos. Isso, não reflete, necessariamente, aumento de casos, mas possivelmente maior sensibilização dos profissionais no preenchimento das fichas de notificação (Macedo et al, 2019).

Em 2011, Ministério da Saúde incluiu a violência doméstica, sexual e/ou outras violências como o $45^{\circ}$ evento de notificação compulsória, através da Portaria $\mathrm{n}^{\circ} 104$, estabelecendo fluxos, critérios, responsabilidades e atribuições aos profissionais e serviços de saúde em todo o território nacional (Brasil, 2011), fato este que pode estar relacionado a elevação no número de registros de notificações, conforme mostram os dados.

Apesar do elevado número de casos de violência sexual registrados no SINAN, estima-se que haja subnotificação, tendo em vista que o processo de implementação do sistema de vigilância de violências e acidentes e violências (Viva), implantando em 2009, não ocorreu de modo homogêneo em todo o território brasileiro (Souza et al, 2014).

No que se refere ao perfil das vítimas, a faixa etária de 5 a 9 anos prevaleceu no estudo (62,1\%), demonstrando similitude quando comparados a outras pesquisas, que também identificaram a predominância de crianças com a faixa etária entre 5 e 9 anos. Entretanto, percebe-se que a maioria dos estudos tem como foco o homem como agressor e a mulher como vítima (Platt et al, 2018; Lopez \& Lefevre, 2019; Lira et al 2017). Reforça-se, assim, a necessidade de outros estudos para melhor visualização sobre o tema, confrontando com as questões de gênero, como categoria importante, quando o assunto é violência sexual.

A raça/cor da pele negra/parda e branca concentraram a maior parte das notificações $(44,2 \%$ e $40,4 \%$ respectivamente), corroborando com estudos nacionais e internacionais que demonstram associação entre violência sexual e a cor da pele, destacando-se negros e pardos. Portanto, diante desses achados, e da sua complexidade, ressalta-se a necessidade 
urgente de romper pré-julgamentos de uma possível associação da violência e a cor da pele. Cabe ressaltar que 14,5\% da variável não consta no sistema, o que alerta para uma melhoria da qualidade do preenchimento da ficha de notificação, conforme apontado em outros estudos (Macedo et al, 2019; Silva et al, 2018).

A avaliação da região de residência das crianças submetidas a violência sexual apontou que o maior volume de notificações no período ocorreu nas regiões sudeste (42,5\%) e Sul (23,6\%). Destarte, é preciso considerar que a quantidade de notificações identificadas nestas regiões possivelmente seja resultado de um trabalho integrado, de vigilância e sensibilização dos profissionais em utilizar a ficha de notificação (Macedo et al, 2019).

O local de ocorrência mais apontado nas notificações foi a residência, o qual também é apresentado em outras pesquisas que revelam o ambiente familiar como um espaço para o abuso (Reis, Prata \& Parra, 2018; Blake, 2014; Rech, Agostino \& Demarco, 2018). Cabe salientar que a escola foi o segundo local com maior frequência de violência sexual, 7\%, corroborando com um estudo realizado recentemente que aponta a escola como local de violência sexual (Santos et al, 2018). O tipo de violência sexual mais notificado foi o estupro. Caracterizado como um facilitador para o aparecimento de psicopatologias graves, prejudicando a evolução psicológica, afetiva e social da vítima, os efeitos do abuso na infância podem se manifestar de diversas maneiras, em qualquer idade da vida, inclusive na reprodução na vida adulta (Hohendorff, Habigzang \& Koller, 2013).

Os principais autores da violência sexual contra crianças são indivíduos do sexo masculino, reforçando a relação de dominação existente do forte pelo mais fraco. A identidade hegemônica da masculinidade afirmada pela sociedade, do exercício de poder exercida pelo agressor, pelo uso da força e da virilidade corrobora para que os homens sejam identificados como os principais autores de violência sexual (Oliveira et al, 2014; Trindade et al, 2014; Sanches et al, 2019). Entretanto, é importante ressaltar que, 19,3\% dos casos notificados de violência sexual foram praticados por mulheres. Assim, é imperativo que sejam desenvolvidos estudos com também com abusadoras do sexo feminino.

Os perfis descritos apontam para a dificuldade em dar visibilidade ao problema, principalmente quando a vítima são crianças do sexo masculino. É fundamental o olhar dos profissionais de saúde em prover o cuidado e o preenchimento da ficha de notificação para que a violência sexual não se torne invisível.

\section{Conclusão}

Inúmeras sequelas físicas, emocionais e psicológicas decorrentes da violência sexual, podem tornar-se irreparáveis, manifestar-se na fase adulta, além da reprodução da violência, que corrobora para a sua perpetuação. Frente esse contexto, pode-se compreender a extrema necessidade de evitar os atos violentos, uma vez que suas sequelas atingem dimensões e gravidade expressivas.

Assim, os estudos epidemiológicos busca não apenas no que se refere a magnitude do problema, mas também: (1) planejamento de ações ou políticas públicas voltadas crianças vítimas de violência sexual do sexo masculino; (2) na visibilidade a um público alvo específico (3) no olhar diferenciado dos profissionais em relação a sinais e sintomas que possam estar associados a abuso, em crianças do sexo masculino pois a preferência dos abusadores é por crianças, pela impossibilidade de defesa (4) na realização de pesquisas sobre questões de gênero associadas a violência sexual, considerando, não apenas crianças vítimas de violência sexual, mas também abusadores do sexo feminino.

Como limitações do estudo, ressalta-se que o periodo estudado, compreende-se 2017, não sendo incluidos os demais anos pela falta de dados no sistema. 


\section{Referências}

Blake, M. T. (2014). Characteristics of sexual violence against adolescent girls and adult women. BMC Women's Health.14:15. https://doi.org/10.1186/14726874-14-15

Brasil. (2018). Ministério da Saúde. Secretaria de Atenção à Saúde. Departamento de Ações Programáticas Estratégicas. Política Nacional de Atenção Integral à Saúde da Criança: orientações para implementação / Ministério da Saúde. Secretaria de Atenção à Saúde. Departamento de Ações Programáticas Estratégicas. - Brasília: Ministério da Saúde.

Brasil. (2011). Ministério da Saúde. Portaria n ${ }^{\circ}$ 104, de 25 de janeiro de 2011. Define as terminologias adotadas em legislação nacional, conforme o disposto no Regulamento Sanitário Internacional 2005 (RSI 2005), a relação de doenças, agravos e eventos em saúde pública de notificação compulsória em todo o território nacional e estabelece fluxo, critérios, responsabilidades e atribuições aos profissionais e serviços de saúde.

Carneiro, S. L. M. A.; \& Cabral, M. A. A. (2010) “O silêncio dos inocentes" - abuso sexual intrafamiliar na infância. Revista EPOS.1(1): 1-18. http://pepsic.bvsalud.org/scielo.php?script=sci_arttext\&pid=S2178700X2010000100005\&lng=pt\&nrm=iss

Dong, M., Anda, R. F., Dube, S. R., Giles, W. H., \& Felitti, V. J. (2003). The relationship of exposure to childhood sexual abuse, to other forms of abuse, neglect and household dysfunction during childhood. Child Abuse Negl; 27:625-39.

Ellery, C.; Corrêa, E.; \& Gadelha, G. (2008). Pesquisa sobre Tráfico de Crianças e Adolescentes para Fins de Exploração Sexual no Estado da Bahia. Instituto Winrock Internacional. Salvador.

Gallo, E. A. G.; Mola, C. L., Wehrmeister, F., Gonçalves, H., Kieling, C., \& Murray, J. (2017). Childhood maltreatment preceding depressive disorder at age 18 years: A prospective Brazilian birth cohort study. Journal of Affective Disorders 217: 218-224.

Hohendorff, J. V; Habigzang, L. F; Koller, S. H. (2013). Sexual violence against boys: epidemiological data, characteristics, and consequences. Psicologia USP; 23(2):395-415. http://www.scielo.br/pdf/pusp/v23n2/en_aop0712.pdf

Lira, M. O. S. C.; Rodrigues, V. P; Rodrigues, A. D; Couto, T. M; Gomes, N. P; Diniz, M. F. (2017). Sexual abuse in childhood and its repercussions in adult life. Texto Contexto Enferm; 26(3):e0080016. http://dx.doi.org/10.1590/0104-07072017000080016

López, C. G., Lefevre ,F. (2019). Descubrimiento del abuso del niño: revelación o silencio. Revista Cubana de Salud Pública; 45(1): 118.https://www.medigraphic.com/cgi-bin/new/resumenI.cgi?IDARTICULO=86031

Martins, C. M. G; Jorge, M. H. P. M. (2010). Child abuse: A review of the history and protection policies. Acta Paul Enferm.; 23(3): 4238. . http://dx.doi.org/10.1590/S0103-21002010000300018

Macedo D. M; Foschiera, L. N, Bordini, T. C. P. M, Habigzang L. F, Koller S. H. (2019). Revisão sistemática de estudos sobre registros de violência contra crianças e adolescentes no Brasil. Ciênc. saúde colet; 24 (2): 487-494. https://doi.org/10.1590/1413-81232018242.34132016]

Oliveira, J. R; Costa, C. O. M, Amaral, M. T. R; Santos, C. A; Assis, S. G, Nascimento, O. C. (2014). Violência sexual e coocorrências em crianças e adolescentes: estudo das incidências ao longo de uma década. Cien Saude Colet; 19(3):759-771.

Platt, V. B., Hauschild, D. B., Back, I. C. \& Guedert, J. M. (2018). Sexual violence against children: authors, victims and conseque nces. Ciência \& Saúde Coletiva. 23(4):1019-31. http://dx.doi.org/10.1590/1413-81232018234.11362016.

Reis, D. M., Prata, L.C.G., Parra, C.R. O impacto da violência intrafamiliar no desenvolvimento psíquico infantil. Psicologia.pt. 2018:1-20. http://www.psicologia.pt/artigos/textos/A1253.pdf. Acesso em 12/07/2020.

Rech, N. B, D’Agostini, F. P, Demarco, T. T. Relações abusivas: a violência sexual no contexto intrafamiliar. Anuário Pesquisa E Extensão Unoesc Videira, 4, e20997. https://portalperiodicos.unoesc.edu.br/apeuv/article/view/20997

Rikic, J; Beljan, P; Milosevic, M; Miškulin, I; Miškulin, M; Mujkić, A. (2017). Transgenerational Transmission of Violence among Parents of Preschool Children in Croatia Acta Clin Croat.; 56(3):478-486.

Sanches, L. C; Araújo, G; Rozin, M. R. L; Rauli, P. M. F. (2019). Violência sexual infantil no Brasil: uma questão de saúde pública. Revista Iberoamericana de Bioética; 9:1-13. http://dx.doi.org/10.14422/rib.i09.y2019.003.

Santos, M. J; Mascarenhas, M. D. M; Rodrigues, M. T. P; Monteiro, R. A. (2018). Caracterização da violência sexual contra crianças e adolescentes na escola - Brasil, 2010-2014. Epidemiol. Serv. Saude, Brasília, 27(2):e2017059. https://doi.org/10.5123/S1679-49742018000200010

Silva, L. M. P; Santos, T. M. B; Santiago, S. R. V; Melo, T. Q; Cardoso, M. D (2018). Análise da completude das notificações de violência perpetradas contra crianças. Rev enferm UFPE online; 12(1):91-100.

Souza, C. S; Costa, C. O. M; Assis, S. G; Musse, J. O; Sobrinho, C. N; Amaral, M. T. R. (2014). Sistema de Vigilância de Violências e Acidentes/VIVA e a notificação da violência infanto-juvenil, no Sistema Único de Saúde/SUS de Feira de Santana-Bahia, Brasil. Cien Saude Colet; 19 (3):773-784.

Trindade, L. C; Linhares, S. M. G. M; Vanrell, J. P, Godoy, D, Martins, J. C. A, Barbas, S. M. A. N. (2014). Sexual Violence against children and vulnerability. Rev. Assoc. Med. Bras; 60(1):70-74. 